\title{
Microplate Array Diagonal Gel Electrophoresis for Cohort Studies of Microsatellite Loci
}

BioTechniques 32:1080-1089 (May 2002)

\author{
Xiao-he Chen, Sandra D. \\ O'Dell, and Ian N.M. Day \\ Southampton General Hospital, \\ Southampton, UK
}

\section{INTRODUCTION}

The identification of short tandem repeat (STR) polymorphisms (22) allowed the use of markers with multiple alleles and high heterozygosities that could be genotyped using PCR. Tetranucleotide repeat markers now have a prominent place in extensive maps that have been constructed for linkage and genome-wide association studies $(19,20)$. Those on the Y-chromosome provide the best model system for dating modern human origins and population diversities by paternal lineage and for studies in paternity testing, forensic casework, and population evolution $(6,9,17)$. Here we describe a utilitarian method for genotyping of tri- and tetranucleotide polymorphisms, using standard laboratory equipment. It is designed to enable the high throughput demanded particularly by population association studies, where greater sample parallelism of analysis for many subjects for single loci may be required, but would also be viable as an alternative format for large-scale linkage studies.

Our new approach derives from methodology we developed to size the fifteen INS gene $5^{\prime}$ VNTR minisatellite class I alleles (641-843 bp) (14). We combine the 96-well microplate array diagonal gel electrophoresis (MADGE) system (1) with a Duracryl ${ }^{\mathrm{TM}}$ polyacrylamide matrix and electrophoretic conditions that permit resolution of alleles over a short $(43.5-\mathrm{mm})$ track length. The method has been tested on three microsatellites. The tetranucleotide repeat (TCAT) $)_{\mathrm{n}}$ in intron 1 of the tyrosine hydroxylase $(T H)$ gene, designated HUMTHO1, has five alleles originally sized as $244,248,252,256$, and $259 \mathrm{bp}$ (15). HUMTHO1 alleles are now referred to by the number of repeats that they contain: $6,7,8,9$, or 9.3 ; the latter allele with 10 repeat units usually has a deleted base in the last unit (16). We have also tested the system in sizing the single copies of two Y-chromosome microsatellites, DYS390, a tetranucleotide repeat, and DYS392, a trinucleotide repeat (8). DYS390 has eight alleles, of which four $(208,212,216$, and $220 \mathrm{bp})$ are common. DYS392 also has eight alleles, and four of these are common: 216, 219, 222, and $225 \mathrm{bp}$. The three markers have been typed in separate cohorts of 2623 and 691 samples.

\section{MATERIALS AND METHODS}

\section{DNA Samples}

Samples of 2623 males from the Northwick Park heart study II (12) and of 691 males and females drawn from the Hertfordshire cohort (7) were used as templates. Y-chromosome markers were genotyped in the former and HUMTHOI in both.

\section{PCR}

HUMTH01. The polymorphism was typed using the PCR conditions described by Weber and May (22) and primers described by Polymeropoulos et al. (15).

Y-chromosome microsatellites. Primers for DYS390 were obtained from http://www.medfac.leidenuniv.nl/ fldo (based on GenBank ${ }^{\circledR}$ accession no. G09611). The sequences of the 
primers for DYS390 were as follows: forward primer, 5'-TATATTTTACACATTTTTGGGCC-3'; reverse primer, 5'-TGACAGTAAAATGAACACATTGC-3'. PCR thermal cycling conditions were 1 cycle at $95^{\circ} \mathrm{C}$ for $3 \mathrm{~min}$ and then $94^{\circ} \mathrm{C}$ for $40 \mathrm{~s}, 56^{\circ} \mathrm{C}$ for $40 \mathrm{~s}$, and $72^{\circ} \mathrm{C}$ for $40 \mathrm{~s}$ for 35 cycles, with a final extension at $72^{\circ} \mathrm{C}$ for $5 \mathrm{~min}$. The sequences of the primers for DYS392 were based on the GenBank accession no. G09867 sequence as follows: forward primer, 5'-GACACCTAAAA GCCAAGAAGGAAAAC-3'; reverse primer, 5'-GAGCGAATACTTAGACCCAGTTGATG-3'. PCR thermal cycling conditions were 1 cycle at $95^{\circ} \mathrm{C}$ for $2 \mathrm{~min}$ and then $94^{\circ} \mathrm{C}$ for $1 \mathrm{~min}$, $58^{\circ} \mathrm{C}$ for $1 \mathrm{~min}$, and $72^{\circ} \mathrm{C}$ for $1 \mathrm{~min}$ for 35 cycles, with a final extension at $72^{\circ} \mathrm{C}$ for $3 \mathrm{~min}$.

Fragment sizing markers. The fragment sizing markers were pairs of PCR products generated from the $I G F 2$ gene, which bracket the allele size ranges of each microsatellite. Templates were extracted from blood of anonymous volunteers. For HUMTHO1, sizing markers were 213 and $291 \mathrm{bp}$. The 213-bp marker forward primer was 5'-GTGCCCCGCCTCCCCGAA-3', and the reverse primer was $5^{\prime}$-GTTTTAA GAGGGTTGTTGTG-3'. For the 291bp marker, the forward primer was 5'-TCACGCCACTTCTCTCCCGC-3', and the reverse primer was $5^{\prime}-\mathrm{TGCTG}$ ACCTAGGAGTGTGCT-3'. PCR thermal cycling conditions for both HUMTHO1 sizing markers were $1 \mathrm{cy}$ cle at $95^{\circ} \mathrm{C}$ for $5 \mathrm{~min}$ and then $92^{\circ} \mathrm{C}$ for $1 \mathrm{~min}, 55^{\circ} \mathrm{C}$ for $1 \mathrm{~min}$, and $72^{\circ} \mathrm{C}$ for $2 \mathrm{~min}$, for 30 cycles. For both Y-chromosome microsatellites, sizing markers were 189 and 253 bp. The 189-bp marker forward primer was 5'-AGTTGTGGAATCGGAAGTGG-3', and the reverse primer was $5^{\prime}$-TAATGCCCGA CCTGAAGATC-3'. The 253-bp marker forward primer was $5^{\prime}$-CAGGAA AGCGACCGGGCATT-3', and reverse primer was 5'-AGGGGCGCAGAG GCGGAGGG3'. PCR thermal cycling conditions for both Y-chromosome microsatellite sizing markers were the same as that for DYS390, except that the annealing temperature was $55^{\circ} \mathrm{C}$.

All reactions were performed in 96well Omniplates (Hybaid, Teddington, $\mathrm{UK}$ ), each $10 \mu \mathrm{L}$ reaction containing
0.4 ng DNA, $50 \mathrm{mM} \mathrm{KCl,} 10 \mathrm{mM}$ Tris $\mathrm{HCl}, \mathrm{pH} 8.3,0.05 \% \mathrm{~W} 1,200 \mu \mathrm{M}$ each dNTP, 4 pmol each primer (MWGBiotech UK, Milton Keynes, UK) and 0.1 U Taq DNA polymerase (Invitrogen, Paisley, UK). The $\mathrm{MgCl}_{2}$ concentration was $2 \mathrm{mM}$ for all PCRs except that of the 253-bp marker, for which it was $1.5 \mathrm{mM}$.

\section{Electrophoresis}

The gel used was a modification of the original MADGE format (1). The open-faced polyacrylamide gel has well spacing that preserves the $8 \times 12$ array and 9-mm pitch of industry-standard microplates. The array is orientated so that electrophoresis proceeds from top to bottom of the horizontal gel and tracks follow a $78.7^{\circ}$ diagonal between wells, providing an available track length of $43.5 \mathrm{~mm}$. Gel formers are available from MadgeBio Ltd. (http://www.madgebio.freeserve.co.uk). A gel consisting of 5\% Duracryl matrix (Genomic Solutions, Ann Arbor, MI, USA) in 1× TAE buffer (11) was used. This mechanically strong and elastic acrylamide-based matrix improved resolution in comparison with standard polyacrylamide. To $50 \mathrm{~mL}$ gel mixture, $150 \mu \mathrm{L}$ each TEMED and $25 \%$ ammonium persulfate were added as polymerization catalysts.

The gel was equilibrated in $1 \times$ TAE buffer containing $5 \mathrm{mM} \mathrm{KCl}$ for $1 \mathrm{~h}$ before use, with one change, to help equalize local ionic conditions near the anode and cathode, which otherwise become imbalanced through differential mobilities of different ions during the run (10). Delaying the start of electrophoresis by $5 \mathrm{~min}$ to reduce the ionic imbalance between sample and gel by diffusion also aided resolution (21). Each $4 \mu \mathrm{L}$ sample prepared for loading consisted of $1 \mu \mathrm{L}$ formamide dye mixture, $1 \mu \mathrm{L} 4 \times$ TAE buffer, $1 \mu \mathrm{L} \mathrm{1:1}$ (v/v) two sizing marker PCR products, and $1 \mu \mathrm{L}$ microsatellite PCR product. A loading buffer was made first, consisting of dye, $4 \times \mathrm{TAE}$, and sizing markers 1:1:1 by volume. One microliter of PCR product was added to $3 \mu \mathrm{L}$ loading buffer, and $2 \mu \mathrm{L}$ were then loaded onto the horizontal gel overlaid with a film of $5 \mathrm{mM} \mathrm{KCl}$ in $1 \times \mathrm{TAE}$, using a 96 split-pin passive transfer de- 


\section{Research Report}

vice (V\&P Scientific, San Diego, CA, USA). A second glass plate was then slid carefully over the surface and secured in position with two longitudinally placed rubber bands. Distortion of bands at the edge of the gel due to ionic effects between gel and buffer was eliminated by inserting a silicone tubing gasket down each side of the gel, leaving the anode and cathode ends unsealed. The gel "sandwich" was then lowered into the electrophoresis tank which contained $1 \times$ TAE buffer preequilibrated to a temperature of $40^{\circ} \mathrm{C}$. Up to 10 loaded gels can be stacked in a rack in the tank. [The tank is manufactured by Ingeny International BV (Goes, The Netherlands), and the rack purpose-built by Ingeny or in house.] Electrophoresis was at a constant voltage of $150 \mathrm{~V}(10 \mathrm{~V} / \mathrm{cm})$ and current of $0.5 \mathrm{~A}$ for $90 \mathrm{~min}$, during which time the temperature of the buffer was thermostatically controlled at $40^{\circ} \mathrm{C}$.

\section{Gel Scanning and Image Analysis}

After electrophoresis, the glass plate covering the gel was removed and the gel was stained in Vistra Green ${ }^{\mathrm{TM}}$ dye (Amersham Biosciences, Little Chalfont, UK). The wet gel was then scanned on a Fluorimager ${ }^{\circledR} 595$ (Molecular Dynamics, Sunnyvale, CA, USA) as described previously (14). The images were viewed and analyzed for fragment size using ImageQuant ${ }^{\mathrm{TM}}$ fragment analysis software (Molecular Dynamics). PCR fragments were sized with reference to two marker bands, which span the allele size ranges. Gel tracks were extracted for analysis using Phoretix $^{\mathrm{TM}}$ software [Phoretix 1D Advanced, version 4.1; NonLinear Dynamics Ltd (http://www.phoretix.com)], as described previously (13). Measurements generated by the Phoretix software were transferred to Microsoft ${ }^{\circledR}$ Excel $^{\circledR}$ for PCR fragment size computation.

For HUMTH01, the determination of PCR fragment sizes was by the following formula:

$$
U=\frac{213}{\frac{213}{291}\left[\left(d^{\prime} 213-d^{\prime} U\right) / d^{\prime} 213\right]}
$$

where $\mathrm{U}$ is the unknown fragment size in $b p, d^{\prime} 213$ is the distance between the 213-bp and 291-bp bands, and d'U is the distance between the unknown fragment band and the 291-bp band. Determination of the Y-chromosome microsatellite fragment size was by the same formula but with different sizing marker values substituted. This formula assumes a log-linear relationship of size (bp) and mobility.

\section{RESULTS AND DISCUSSION}

Electrophoresis of $96 \mathrm{HUMTHOI}$ PCR products from the Northwick Park Heart Study II cohort (NPHSII) (12) on a 5\% Duracryl MADGE gel is shown in Figure 1. Figure $2 \mathrm{a}$ shows images of individual tracks representing the 15 different allelic combinations generated by the five alleles at the HUMTHO1 lo-

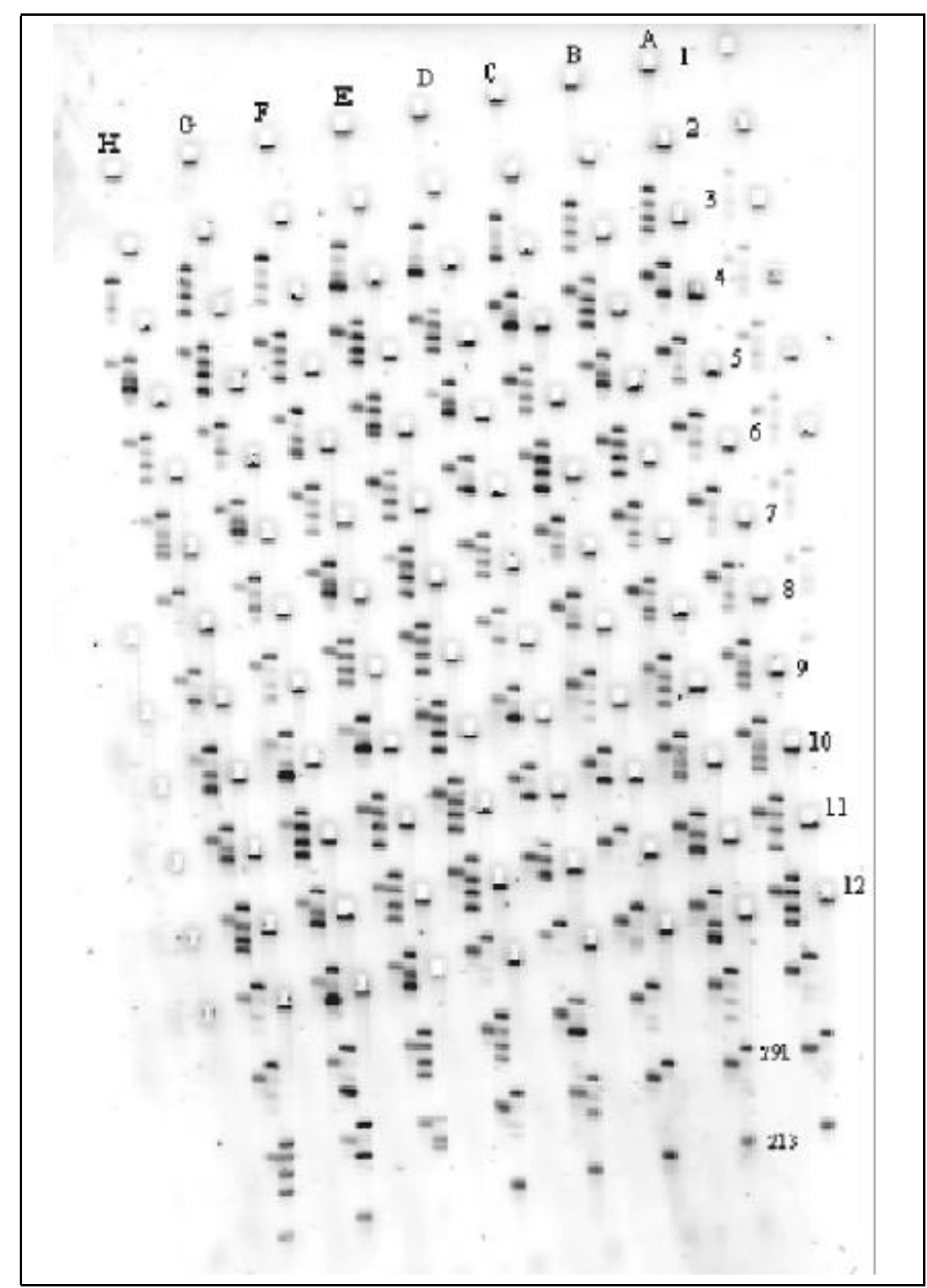

Figure 1. Electrophoresis of HUMTH01 tetranucleotide fragments on a 5\% Duracryl MADGE gel. The wells are in an $8 \times 12$ Omniplate configuration. The direction of electrophoresis is from top to bottom, in tracks following a $78.7^{\circ}$ diagonal between wells, giving a maximum available diagonal track length of $43.5 \mathrm{~mm}$ between wells. The internal size markers 213 and $291 \mathrm{bp}$ are run in each track. A single band denotes a homozygote, and heterozygotes appear as two allele bands and one or two heteroduplex bands between the 213- and 291-bp limits, which are marked in track B12. 


\section{Research Report}

A

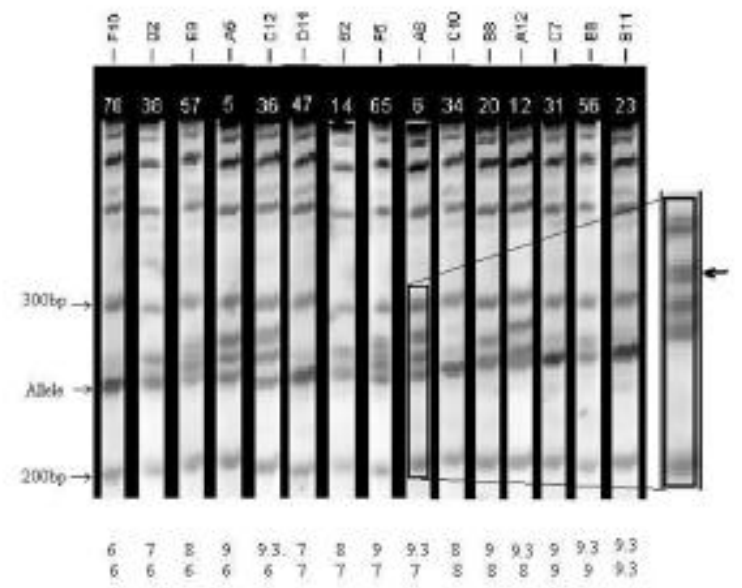

B
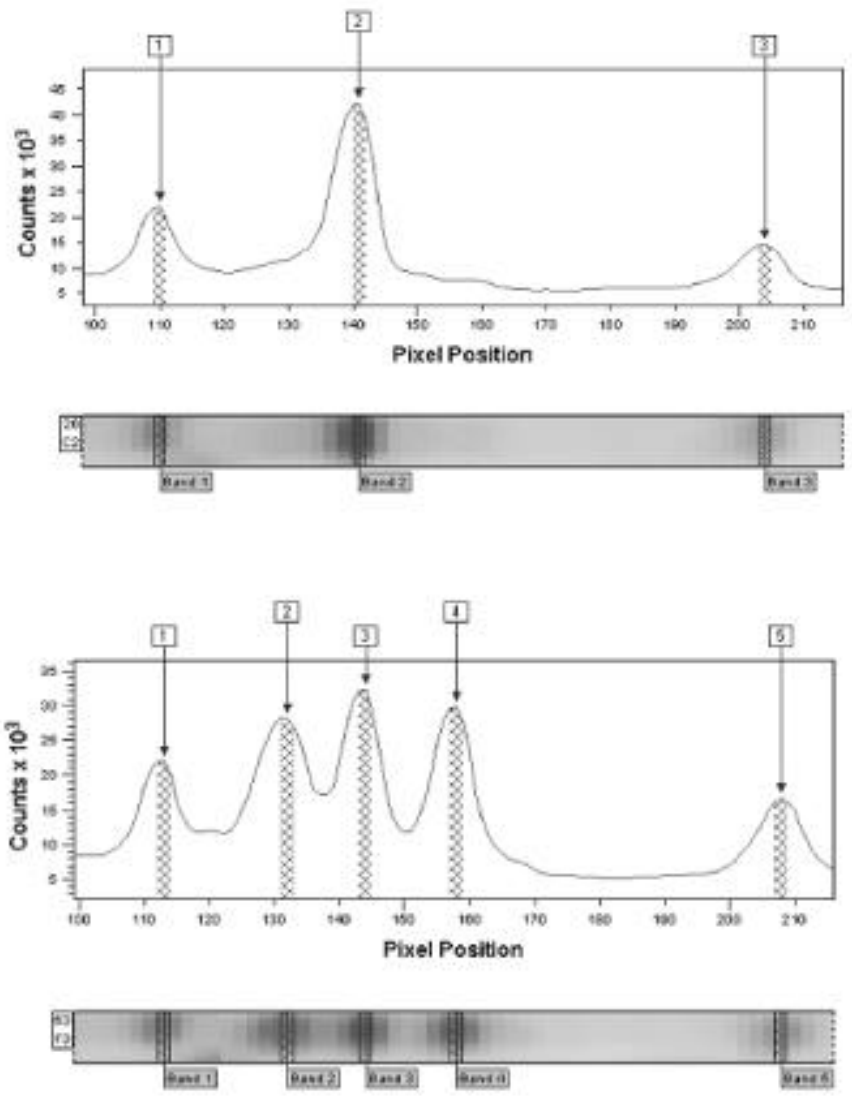

Figure 2. Images and electrophoretograms of individual MADGE gel tracks. (a) Fifteen selected tracks derived from a gel similar to that shown in Figure 1 illustrate the 15 genotypes produced by the five alleles at the HUMTHOI locus. Images are generated by Phoretix software. Here the PCR products were co-run with a 100-bp ladder (Promega, Madison, WI, USA), and homo- and heteroduplex bands are spanned by the 200- and 300-bp marker bands in each track. In the inset panel, an arrow indicates the heteroduplex band generated by a 2.3 -bp repeat difference in allele size. Genotypes are indicated below each track, with numbers representing the alleles 6,7, 8,9, and 9.3. (b) These electrophoretograms are generated by Phoretix software. The upper panel shows a single track representing a HUMTHOI homozygote. The homoduplex is band 2 . The lower panel shows a single track representing a heterozygote. The corunning heteroduplexes are in band 2, and the two homoduplex alleles are bands 3 and 4 . Bands 1 and 3 in the upper panel and bands 1 and 5 in the lower panel are the sizing marker bands 213 and $291 \mathrm{bp}$. 
cus. The electrophoretograms in Figure $2 \mathrm{~b}$ illustrate the fluorescence intensity of each band in a single track, as it is scanned by the laser beam of the Fluorimager. The intensity patterns in two tracks are shown, with the band representing the shortest fragment on the right side of the figure. The calculated sizes of the two HUMTHOI fragments represented by homoduplex bands are given in the figure legend. The other bands in the electrophoretogram represent the flanking size markers, 213 and $291 \mathrm{bp}$, and the heteroduplex bands that appear under native conditions. In many cases, the combined pattern in tracks can enable direct visual recognition of genotype, but the main focus is the two homoduplex bands and bracketing size markers. Fragments sized in 691 individuals from the Hertfordshire cohort and arbitrarily binned to five groups representing the published alleles 6, 7, 8, 9, and 9.3 are shown in Figure 3. All fragments fell into distinct "bins" defining designated allele size. The distribution of the 1382 binned alleles confirmed previous observations that alleles 6-9 are composed of 4-bp repeats, with allele 9.3 including a 3-bp unit (16). A total of 2463 DYS390 and 2623 DYS392 alleles from the NPHSII cohort were analyzed. In both microsatellites, fragments were sized and arbitrarily binned to eight distinct groups, which defined the allele sizes. Binning of the trinucleotide repeat DYS392 PCR fragments is shown in Figure 4. The median fragment size in each bin coincides with allele sizes that were

Table 1. Allele Frequency Distribution of HUMTH01 in Hertfordshire Cohort

\begin{tabular}{|ccrcc|}
\hline $\begin{array}{c}\text { Allele bin } \\
\text { (bp) }\end{array}$ & \multicolumn{2}{c}{ Frequency } & & \\
\cline { 2 - 3 }$(\boldsymbol{n})$ & $\mathbf{( \% )}$ & Mean & SD \\
\hline 246 & 312 & 22.58 & 246.6 & 0.92 \\
250 & 250 & 18.09 & 250.6 & 0.95 \\
254 & 131 & 9.48 & 254.2 & 0.63 \\
258 & 171 & 12.37 & 258.0 & 0.88 \\
261 & 518 & 37.48 & 261.8 & 0.93 \\
Total & 1382 & 100.0 & & \\
\hline
\end{tabular}

Table 2. Allele Frequency Distribution of DYS392 in NPHSII Cohort

\begin{tabular}{|crrrr|}
\hline $\begin{array}{c}\text { Allele bin } \\
\text { (bp) }\end{array}$ & \multicolumn{2}{c}{ Frequency } & & \\
\cline { 2 - 4 }$(\boldsymbol{n})$ & $(\%)$ & Mean & SD \\
\hline 210 & 6 & 0.23 & 209.9 & 0.19 \\
213 & 14 & 0.53 & 213.3 & 0.30 \\
216 & 670 & 24.54 & 216.1 & 0.88 \\
219 & 187 & 7.13 & 218.9 & 0.65 \\
222 & 1534 & 58.48 & 222.1 & 0.91 \\
225 & 196 & 7.47 & 225.4 & 0.65 \\
228 & 13 & 0.50 & 228.3 & 0.45 \\
231 & 3 & 0.11 & 230.9 & 0.13 \\
Total & 2623 & 100.0 & & \\
\hline
\end{tabular}

predicted from GenBank sequence.

Precision of binning (i.e., recognition of distinct subsets of the discrete sets of alleles represented by the histogram blocks) is possible given the size gap between each cluster and is a reasonable test of assay quality in large population studies. Our method is utilitarian, maximizing resolution on very short gel tracks to achieve convenient sample parallelism and direct microplate compatibility. Alleles should fall into discrete groups in frequency histograms by size (to nearest estimated 0.1 nucleotide), representing each repeat length evident in the population (e.g., $x, x+4)$. Visual inspection of Figures 3 and 4 confirms that discrete bins are evident. Such cluster analysis forms a standard "bootstrap" for microsatellite allele binning. It is based on the assumption that only particular genotypes exist (e.g., AA, Aa, aa for SNPs, or $x$, $x+4, x+8 \ldots x+4 n$ for a tetranucleotide

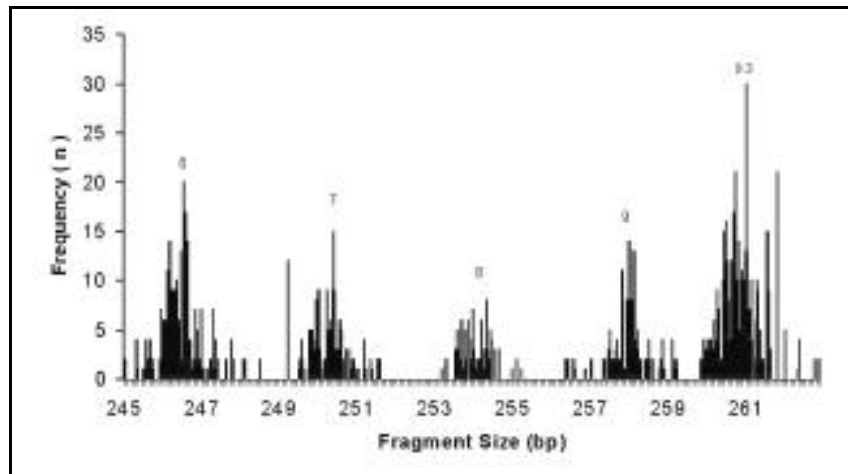

Figure 3. Frequency of computed HUMTH01 fragment sizes from 691 individuals. Fragment sizes were computed from the gel image using the formula given in the text and are correct to one decimal place of nucleotide. All fragments except the 252-bp one fall into five distinct groups ("bins") defining the alleles $6,7,8,9$, and 9.3 , identified on the chart.

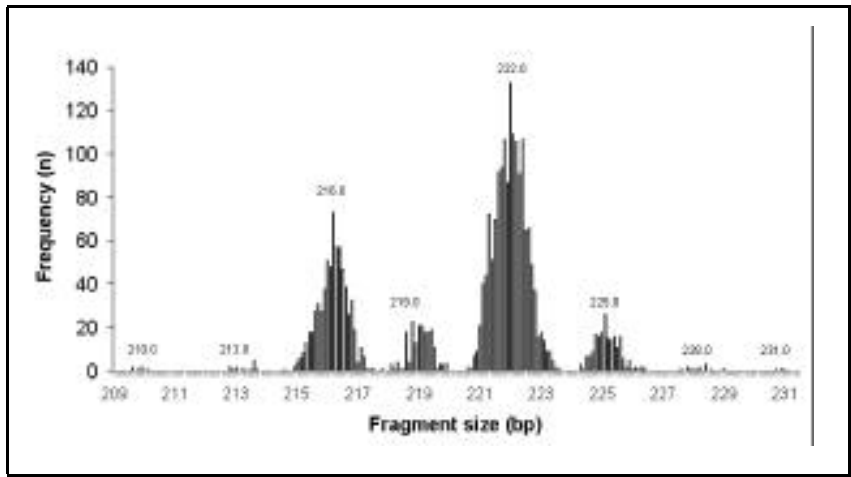

Figure 4. Frequency of computed DYS392 fragment sizes from 2623 individuals. All fragments amplified from this trinucleotide microsatellite fall into eight distinct groups ("bins") defining the allele sizes, 3 bp apart, of which four are common. The numbers indicate the size of the allele representing the median of each bin range. 
repeat). The assumption fails if rare alleles exist. HUMTHO1 allele 10, with one base pair more than allele 9.3 is present at approximately $1 \%$ in Caucasians (4). Our method would not distinguish these two alleles, although the disruption of the typical bell curve of estimated allele size evident for allele 9.3 might raise suspicion of heterogeneity.

For the two microsatellites illustrated, we have estimated the mean size and standard deviation (in bp) for each allele bin created (Tables 1 and 2). Betweenbin cutpoints were at frequency minima. For the four common alleles of DYS392, the average standard deviation is 0.77 bp. For the five HUMTHO1 alleles identified, the average standard deviation is $0.87 \mathrm{bp}$. This gives 3.9 standard deviation between mean allele sizes for the trinucleotide repeat DYS392 and 4.6 standard deviation between means for the predominantly tetranucleotide repeat
HUMTH01. Assuming a normal distribution (most allele bins show bell shape of frequency against size estimated to nearest $0.1 \mathrm{bp}$, although there are gaps between most bins suggesting truncated distributions), $5 \%$ of samples in one bin would fall outside cutpoints placed 2 standard deviations above and below the mean for that bin. If all calls were made (based on arbitrary cutpoints at minima between frequency peaks), then there would be, typically, approximately a 95\% correct call rate. Relative to very high-resolution long tracks (slab or capillary), this represents a high error rate but has utilitarian value for large-scale association studies. Exclusion of calling for a small window of sizes near the frequency minima will considerably reduce error rate (e.g., to 99\% correct). The specific examples used represent large microsatellite products and for loci or products in which size differences are relatively greater (e.g., 153, 156, and 159 bp), confidence limits will be improved.

We compared allele frequencies obtained by the MADGE method with others reported in Caucasian samples. In 691 individuals from our Hertfordshire cohort, frequencies of the five HUMTHO1 allele sizes were as shown in Table 1. Genotypes represented no significant deviation from Hardy Weinberg equilibrium (Chi square test $P=$ 0.103 ; df $=14$ ). The corresponding allele frequencies originally reported by Polymeropoulos et al. (15) were $26 \%$ (allele 6), 14\% (allele 7), 13\% (allele 8), $26 \%$ (allele 9), and 21\% (allele 9.3) in only 70 chromosomes of unrelated individuals, using polyacrylamide gel electrophoresis of PCR products generated with ${ }^{32} \mathrm{P}$-labeled primers followed by autoradiography. Sharma et al. (18) recorded allele frequencies of $24 \%$, $17 \%, 13 \%, 20 \%$, and $26 \%$, respective- 
ly, in 206 subjects, using ABI Genescan $^{\mathrm{TM}}$ (Applied Biosystems, Foster City, CA, USA).

Our distribution of allele frequencies for tetranucleotide microsatellite DYS390 is in close agreement with those reported in other European populations (2) http://www.medfac.leidenuniv.nl/fldo). In $2460 \mathrm{Y}$-chromosomes in the NPHSII cohort, the four common alleles 208, 212, 216, and 220 bp accounted for $12.1 \%, 28.2 \%, 45.7 \%$, and $12.8 \%$, respectively, of total alleles. The corresponding frequencies reported in European populations were $13.4 \%, 28.1 \%, 37.7 \%$, and $14.1 \%$. The distribution of allele frequencies for DYS392 is in agreement with that in German (5) and Dutch (3) populations. In 2623 Y-chromosomes in the NPHSII cohort, of the four common alleles 216 , 219, 222, and 225 bp, 222 bp accounted for $58.5 \%$ of the total (Table 2). It represents $56 \%$ in the German (5) and $55 \%$ in the Dutch (3) populations.

This method is presented as a utilitarian approach for the sizing of microsatellite alleles in large numbers of samples precisely, rapidly, and at low cost. Our principal concern has been to create high precision in the interpolation of estimated PCR fragment sizes to achieve precise binning, not to obtain accurate absolute sizing. However, we have checked the actual size of representative fragments from allele bins by direct sequencing. We have also undertaken repeat runs of the same subjects as a further evaluation of precision and can confirm that binning works as well between as within gels. Our system lacks the single-nucleotide resolution required for the detection of rare subrepeat alleles demanded by forensic testing; however, the chief advantages of the MADGE method lie in low-cost reagents, the use of standard laboratory equipment, and a format that should be particularly suited to population studies of selected single markers.

\section{ACKNOWLEDGMENTS}

This work was funded by the Wessex Medical Trust and UK Medical Research Council. Dr. G.J. Miller is thanked for permission to access the Northwick Park Heart Study II DNA bank.

\section{REFERENCES}

1.Day, I.N.M. and S.E. Humphries. 1994. Electrophoresis for genotyping: microplate array diagonal gel electrophoresis on horizontal polyacrylamide gels, Hydrolink or agarose. Anal. Biochem. 222:391-394.

2.de Knijff, P., M. Kayser, A. Caglia, D. Corach, N. Fretwell, C. Gehrig, G. Graziosi, F. Heidorn, et al. 1997. Chromosome Y microsatellites: population genetic and evolutionary aspects. Int. J. Legal Med. 110:134-149.

3.de Knijff, P. 2000. Messages through bottlenecks: on the combined use of slow and fast evolving polymorphic markers on the human Y chromosome. Am. J. Hum. Genet. 67:10551061.

4.Evett, I.W., P.D. Gill, J.A. Lambert, N. OIdroyd, R. Frazier, S. Watson, S. Panchal, A. Connolly, and C. Kimpton. 1997. Statistical analysis of data for three British ethnic groups from a new STR multiplex. Int. J. Legal Med. 110:5-9.

5.Forster, P., A. Rohl, P. Lunnemann, C. Brinkmann, T. Zerjal, C. Tyler-Smith, and B. Brinkmann. 2000. A short tandem repeatbased phylogeny for the human Y-chromosome. Am. J. Hum. Genet. 67:182-196.

6.Gill, P., C. Brenner, B. Brinkmann, B. Budowle, A. Carracedo, M.A. Jobling, P. de Knijff, M. Kayser, et al. 2001. DNA commission of the International Society of Forensic Genetics: recommendations on forensic analysis using Y-chromosome STRs. Int. J. Legal Med. 114:305-309.

7.Henry, J.A., M. Bolla, C. Osmond, C. Fall, D.J.P. Barker, and S.E. Humphries. 1997. The effects of genotype and infant weight on adult plasma levels of fibrinogen, factor VII, and LDL cholesterol are additive. J. Med. Genet. 34:553-558.

8.Kayser, M., A. Caglia, D. Corach, N. Fretwell, C. Gehrig, G. Graziosi, F. Heidorn, S. Herrmann, et al. 1997. Evaluation of Y-chromosomal STRs: a multicenter study. Int. J. Legal Med. 110:125-149.

9.Kayser, M., L. Roewer, M. Hedman, L. Henke, J. Henke, S. Brauer, C. Kruger, M. Krawczak, et al. 2000. Characteristics and frequency of germline mutations at microsatellite loci from the human $\mathrm{Y}$ chromosome, as revealed by direct observation in father/son pairs. Am. J. Hum. Genet. 66:1580-1588.

10.Kozulic, B. 1994. Looking at bands from another side. Anal. Biochem. 216:253-261.

11.Maniatis, T., E.F. Frisch, and J. Sambrook. 1982. Molecular Cloning: A Laboratory Manual. CSH Laboratory Press, Cold Spring Harbor, NY.

12.Miller, G.J., K.A. Bauer, S. Barzegar, J.A. Cooper, and R.D. Rosenberg. 1996. Increased activation of the haemostatic system in men at high risk of fatal coronary heart disease. Thromb. Haemost. 75:767-771.

13.O'Dell, S.D., T.R. Gaunt, and I.N.M. Day. 2000. SNP genotyping by combination of 192well MADGE, ARMS and computerised gel image analysis. BioTechniques 29:500-506.

14.O'Dell, S.D., X. Chen, and I.N.M. Day. 2000. Higher resolution microplate array diagonal gel electrophoresis: application to a multiallelic minisatellite. Hum. Mutat. 15:565-576.
15.Polymeropoulos, M.H., H. Xiao, D.S. Rath, and C.R. Merril. 1991. Tetranucleotide repeat polymorphism at the human tyrosine hydroxylase gene (TH). Nucleic Acids Res. 19:3753.

16.Puers, C., H.A. Hammond, L. Jin, C.T. Caskey, and J.W. Schumm. 1993. Identification of repeat sequence heterogeneity at the polymorphic short tandem repeat locus HUMTH01 (AATG) ${ }_{n}$ and reassignment of alleles in population analysis by using a locus specific allelic ladder. Am. J. Hum. Genet. 53:953-958.

17.Quintana-Murci, L., C. Krausz, and K. McElreavey. 2001.The human Y chromosome: function, evolution and disease. Forensic Sci. Int. 118:169-181.

18.Sharma, P., A. Hingorani, H. Jia, M. Ashby, R. Hopper, D. Clayton, and M. Brown. 1998. Positive association of tyrosine hydroxylase microsatellite marker to essential hypertension. Hypertension 32:676-682.

19.Sheffield, V.C., J.L. Weber, K.H. Buetow, J.C. Murray, D.A. Even, K. Wiles, J.M. Gastier, J.C. Pulido, et al. 1995. A collection of tri- and tetranucleotide repeat markers used to generate high quality, high resolution human genome-wide linkage maps. Hum. Mol. Genet. 4:1837-1844

20.The Utah Marker Development Group. 1995. A collection of ordered tetranuceotide repeat markers from the human genome. Am. J. Hum. Genet. 57:619-628.

21.Voropanov, A.M. and I.N.M. Day. 2000. Elimination of dumbbell bands and enhancement of resolution in MADGE using delayed start electrophoresis. BioTechniques 28:32-34.

22.Weber, J.L. and P.E. May. 1989. Abundant class of human DNA polymorphisms which can be typed using the polymerase chain reaction. Am. J. Hum. Genet. 44:338-396.

Received 5 November 2001; accepted 23 January 2002.

Address correspondence to:

Prof. Ian N.M. Day

Division of Human Genetics

University of Southampton School of Medicine Southampton General Hospital

Duthie Building (MP 808), Tremona Road

Southampton S016 6YD, UK

e-mail: inmd@soton.ac.uk

For reprints of this or any other article, contact Reprints@BioTechniques.com 